\section{Crack Resistance in Cherry Tomato Fruit Correlates with Cuticular Membrane Thickness}

\author{
Antonio J. Matas ${ }^{1}$ \\ Department of Plant Biology, Cornell University, Ithaca, NY 14853
}

Eward D. Cobb

Department of Plant Breeding, Cornell University, Ithaca, NY 14853

Dominick J. Paolillo, Jr.

Department of Plant Biology, Cornell University, Ithaca, NY 14853

Karl J. Niklas ${ }^{2}$

Department of Plant Biology, Cornell University, Ithaca, NY 14853

Additional index words. biomechanics, collenchyma, cuticular membrane, fruit cracking, Lycopersicon esculentum, strain hardening

\begin{abstract}
The mechanical properties and anatomy of fruit wall peels and their enzymeisolated cuticular membranes (CM) are reported for three cherry tomato (Lycopersicon esculentum Mill.) cultivars that are crack-resistant, crack-intermediate, and crack-prone (i.e., Inbred 10, Sweet 100, and Sausalito Cocktail, respectively). The resistant and intermediate fruit peels strain-hardened when extended progressively; those of the crack-prone cultivar did so only modestly. The CM of all cultivars strain-hardened when extended with small forces; the $\mathrm{CM}$ of the intermediate and crack-prone cultivars strain-softened under tensile forces that did not strain-soften the crack-resistant cultivar. The peels and $\mathrm{CM}$ of the resistant cultivar were stiffer, stronger, and required more energy to break than crack-prone peels. The $\mathrm{CM}$ of crack-resistant peels developed deeper within the subepidermis than in the crack-prone or crack-intermediate peels. The $\mathrm{CM}$ in the outer epidermal periclinal walls of the crack-resistant and crack-intermediate cultivars was thicker than that of crack-prone peels. These data indicate that CM thickness can be used to gauge crack susceptibility among cherry tomato fruit, which can be useful in breeding programs and would facilitate QTL mapping of the underlying genetic factors.
\end{abstract}

Tomato fruit cracking causes degradation of appearance and subsequent serious economic losses. The susceptibility of cracking has been correlated with a range of environmental and physiological factors as well as ultrastructural and anatomical features (Hankinson and Rao, 1979; Thompson, 2001; Wiedermann and Neinhuis, 1998). However, the factors responsible for fruit cracking remain problematic, although many authors emphasise the role of the cuticular membrane (CM; terminology of Holloway, 1982; Jeffree, 1996) and the extent to which it develops in epidermal and subepidermal walls (e.g., Emmons and Scott, 1997, 1998; Voisey et al., 1970; Wattendorf and Holloway, 1980).

The objective of this study is to determine the extent to which the CM contributes to the ability of tomato fruit to resist tension, both mechanically and anatomically. Because the walls of the epidermis and adjoining subepidermal cells are placed in tension when primary organs are turgid (Kutschera 1989; Niklas and Paolillo, 1997, 1998), the CMlikely

Received for publication 7 Oct. 2003. Accepted for publication 25 Jan. 2004.

${ }^{1}$ Current address Department of Plant Breeding, Estación Experimental La Mayora (CSIC), Algarrobo-Costa 29750, Malaga, Spain.

${ }^{2}$ To whom reprint requests and correspondence should be sent; e-mail kjn2@cornell.edu. functions as a tensile skin whose mechanical properties can be influenced by temperature or external water (Cotner et al., 1969; Petracek and Bukovac, 1995; Round et al., 2000; see also Sekse, 1995).

Here, we report the mechanical properties (i.e., strength, stiffness, and work of fracture) and anatomy of fruit wall peels and their enzyme-isolated CM from three cherry tomato cultivars that differ in their susceptibility to cracking, i.e., Inbred 10 (crack-resistant), Sausalito Cocktail (crack-intermediate), and Sweet 100 (crack-prone). Comparisons between the mechanical behavior of fruit peels and their isolated $\mathrm{CM}$ are used to evaluate the role of

Our data indicate that the ability of fruit to resist cracking is highly correlated with the thickness of the $\mathrm{CM}$ in the outer periclinal epidermal walls and the extent to which the CM develops within subepidermal cell walls. Although differences in chemistry or ultrastructure must account for differences observed in the mechanical behaviour we report for isolated $\mathrm{CM}$, we propose that $\mathrm{CM}$ thickness provides a convenient gauge to evaluate the cracking susceptibility of different cherry tomatoes.

\section{Materials and Methods}

Culture and sampling. Three cherry tomato cultivars differing in their susceptibility to fruit the $\mathrm{CM}$ in intact fruit. cracking were selected for study, i.e., 'Inbred 10, a crack-resistant Cornell line released as CT 95-946, 'Sausalito Cocktail,' a crackintermediate Cornell line released by the Territorial Seed Company (Cottage Grove, Ore.), and 'Sweet 100,' a crack-susceptible cultivar commercially available from Agway.

Four cherry tomato plants per cultivar were grown in pots to maturity (using commercially available seeds and Cornell potting mix) under greenhouse conditions without supplemental lighting. Each plant was irrigated and fertilized on a regular schedule. Flowers were handled daily to facilitate pollination, and each inflorescence was labelled and monitored to evaluate fruit development and ripening. Between 30 and 50 fruit were selected from each cultivar when fully ripe in the sequence of their appearance, i.e., 1 or $2 \mathrm{~d}$ after reaching the breaker stage. Each sample was tested mechanically within $5 \mathrm{~h}$ of harvesting.

Sample preparation. Rectangular paradermal segments of the outer fruit wall (aligned either longitudinally or equatorially with respect to the pedicle-style fruit axis) were removed using two parallel razorblades bonded to a metal block to assure uniform segment width $(5.25 \mathrm{~mm})$ and depth of cut $(230 \pm 35$ $\mu \mathrm{m})$. Segment-length varied due to differences in fruit size and shape.

The outer fruit wall of each sample was then peeled off by hand and placed in a petri dish subepidermal side downward on filter paper hydrated with the liquid and soft pulp of the fruit from which it was removed. This protocol was used to reduce desiccation, maintain tissue osmolarity, and prevent direct wetting of the CM. A small portion of the outer fruit wall next to each segment was also removed and preserved in FAA for anatomical study. Measurements of peel and CM thickness on light micrographs were used to determine the cross sectional areas of samples tested mechanically to compute stresses (see below). Importantly, peel and CM thickness varied across the three cultivars because of the extent to which the CM extended below the outer periclinal walls of the epidermis (see Fig. 3).

Duplicate peels were also removed from each fruit to compare peels with their enzymeisolated CM using the protocol of Orgell (1955) as modified by Yamada et al. (1964) (see Petracek and Bukovac, 1995). Excised samples were suspended in a mixture of cellulase and pectinase of fungal origin $(0.2 \%$ and $2.0 \% \mathrm{w} / \mathrm{v}$, respectively; Sigma catalogue numbers C-1184 and P-4716, respectively) in a sodium citrate buffer (50 mM, pH 4.0) with $1.0 \mathrm{~mm} \mathrm{NaN}_{3}$ (to prevent microbial growth). Suspensions were aspirated to facilitate enzyme penetration before incubation at $35^{\circ} \mathrm{C}$ for 7 to $10 \mathrm{~d}$ during which suspensions were agitated by hand daily. Samples were then rinsed in citrate buffer and inspected microscopically to determine their cross section and appearance before mechanical testing.

Mechanical tests. Peels and their corresponding CM were mounted for mechanical tests using two small metal rods suspended by triangular stirrups between the cross heads of an Instron (model 4502) testing machine 
(Instron Company, Calif.). A small amount of fast-drying super glue was spread over the middle of each rod and the external surface of both ends of each sample. Each end of the sample was then folded over one of the two rods before aligning the two stirrups parallel to the Instron cross-head axis. During tests, samples were continuously hydrated (with the liquid extracted from their fruit) by means of a micropipette applied to the subepidermis. A small piece of hydrated tissue paper was placed on this surface to maximise uniform hydration and further reduce desiccation. In passing, we note that hydration of specimens with water (as opposed to fruit-fluid extracts) or dehydration during testing resulted in data differing substantially from those reported here.

To determine tensile strains, the length of each mounted sample was measured before extension with a hand held microcaliper. The length of the CM exposed between the two folds was also measured. To determine stresses, the tensile force exerted during each test was divided by the average cross sectional area of each sample.

Successive loading-unloading tests were used to determine the affects of repeated stretching-relaxation cycles on the Young's modulus (stiffness) of a sample. Uniaxial tension tests were used to determine breaking stress (strength) and the work of fracture (energy required to rip a sample). Because no sample could be tested both ways, the data from both tests were juxtaposed to determine the mechanical properties of peels and their isolated CM. Unless otherwise noted, a 2.0 $\mathrm{mm} \cdot \mathrm{s}^{-1}$ deformation rate (i.e., cross head displacement rate) was used in all tests. The protocols for each of the two types of tests were as follows.

In successive loading-unloading (cyclical) tests, each peel was increasingly extended (using an increasing sequence of forces from 0.10 to $0.65 \mathrm{~N}$ in $0.05-\mathrm{N}$ increments) and allowed to relax before reloading. Because the $\mathrm{CM}$ contributes only a percentage fraction of the total cross sectional area of a peel, equivalent tensile forces do not generate equivalent stresses in peels and their isolated $\mathrm{CM}$. Therefore, the decimal fraction $F$ of the total peel cross section occupied by the $\mathrm{CM}$ was determined and used to compute the tensile forces required to produce equivalent stresses in the CM using the formula $P_{\mathrm{e}}=F P_{\mathrm{p}}$, where $P_{\mathrm{e}}$ is the equivalent stress force and $P_{\mathrm{p}}$ is the force used to extend a peel. For convenience, data from loading-unloading tests of the $\mathrm{CM}$ are plotted as a function of $P$

Uniaxial tensile tests were performed using the aforementioned protocol. However, each sample was tested to failure to determine the breaking stress and the breaking strain. Uniaxial tensile tests were also used to determine the work of fracture $W$ (the energy per unit cross sectional area required to propagate a crack) by making a small cut of known length on one side of a sample before it was tested to failure. The Young's modulus $E$ and the breaking stress $\sigma_{\mathrm{b}}$ of each sample were then used to compute $W$ using the formula $W=\pi_{\mathrm{b}}{ }^{2} / 2 E$ (see Denny, 1988; Niklas, 1992).
Anatomical protocols. Materials to be examined histologically were washed in $50 \%$ ethanol, rehydrated, and sectioned at a thickness of $10 \mu \mathrm{m}$, frozen in water and using a cryostat. The sections were observed unstained or stained in zinc-chlor-iodine for detection of cellulosic walls (Peacock, 1966). Unstained sections were also observed between crossed polarizers, with and without the insertion of a rose plate, which develops a color image that allows for the determination of the net orientation of unstained cellulose wall microfibrils (Bennett, 1950). Stained sections were also examined using a single polarizing filter beneath the specimen and a rotating stage to detect dichroism, which allows the determination of the net orientation of stained cellulose wall fibrils (Roelofsen, 1959). Photographs were obtained using an image capture program under the control of a microcomputer.
Results

Mechanical tests indicated that the fruit walls and $\mathrm{CM}$ of each cultivar are isotropic, i.e., their mechanical responses were independent of the direction of applied tensile tests. Therefore, for convenience, the data presented here are from tests using longitudinally oriented peels and enzyme-isolated CM.

The peels of all three cultivars strain-hardened (increased in stiffness) when repeatedly extended with increasing loads and allowed to relax. The relationship between the Young's modulus (stiffness) and the tensile load for each cultivar was log-log linear (Fig. 1A). Among the three cultivars, the peels from the crack-resistant cultivar strain-hardened more rapidly than those from the crack-intermediate or crack-prone cultivars as gauged by the slopes of $\log$-log regression curves. For comparatively small tensile loads (i.e., $0.1 \mathrm{~N} \leq P \leq 0.4 \mathrm{~N}$ ),
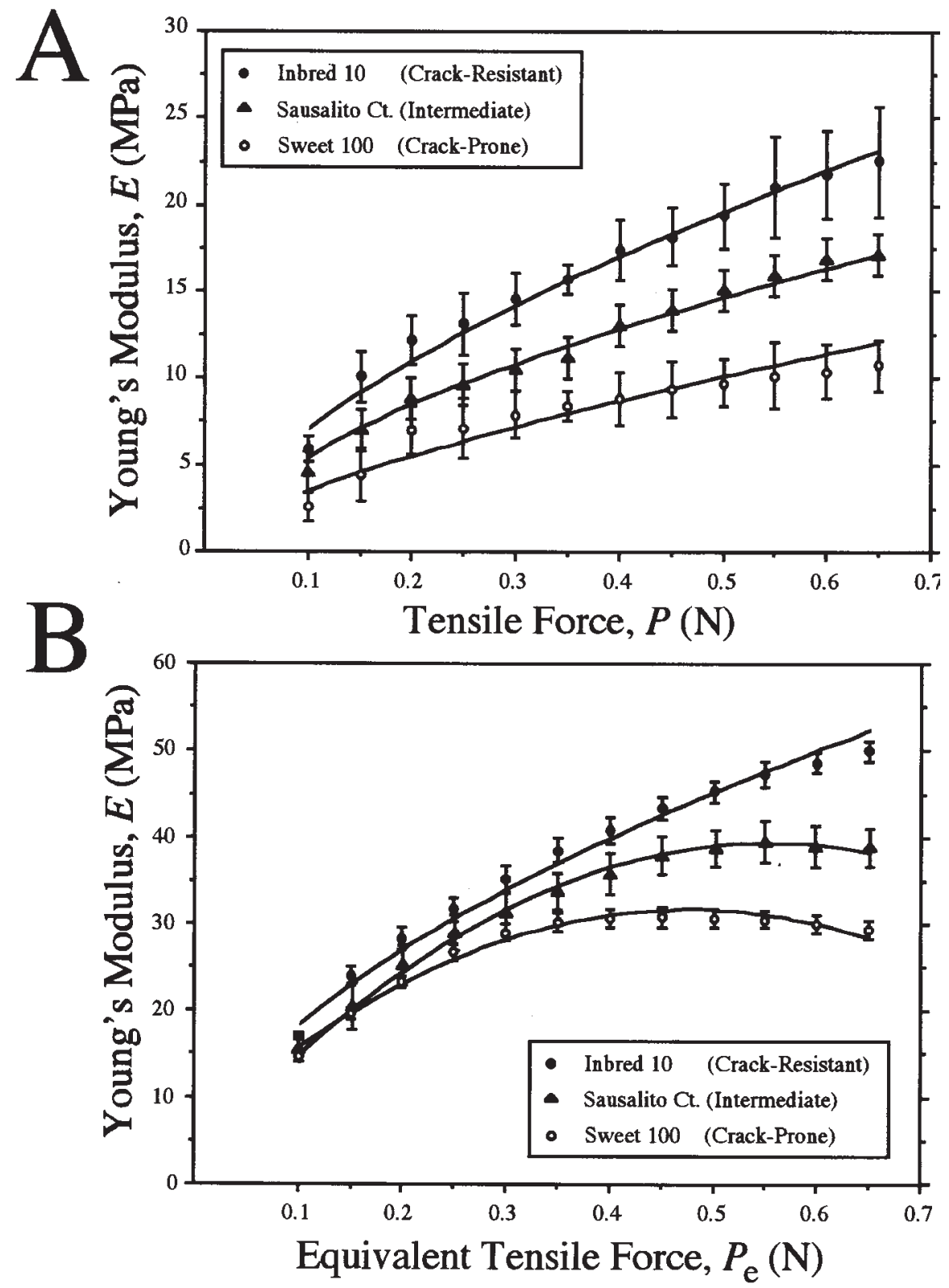

Fig. 1. Young's modulus (stiffness measured in tension) of fruit peels (A) and their isolated cuticular membranes (B) when repeatedly stretched and allowed to relax (successive loading-unloading cycles). Solid lines are regression curves (log-log linear in A; second-order polynomials in B). Across regression analyses, $0.92 \leq r^{2} \leq 0.98, n=12$ means based on 10 samples each). 
the differences among the Young's moduli of peels were not statistically significant among the three cultivars. For larger loads (i.e., $P>$ $0.4 \mathrm{~N}$ ), however, the differences among the moduli between the crack-prone cultivar and either the crack-intermediate or crack-resistant cultivar were statistically significant $(p<$ 0.0001) (Fig. 1A).

The behavior of the isolated CM from the crack-resistant cultivar differed from that of the other two cultivars, especially under large equivalent loads. Specifically, the crack-resistant cultivar CM did not strain-soften, whereas the CM from the other cultivars did for $P_{e} \geq$ $0.5 \mathrm{~N}$ (Fig. 1B). The CM of the crack-prone cultivar strain-hardened the least and began to strain-soften more rapidly as gauged by the slopes of second order polynomial regression curves.

Tensile tests to mechanical failure indicated that the Young's modulus of peels from the crack-resistant cultivar and its isolated $\mathrm{CM}$ was statistically significantly greater than that of the crack-prone cultivar. Pair-wise comparisons of mean Young's moduli indicated that the stiffness of the peels and CM of the intermediate cultivar were indistinguishable from those of either the crack-resistant or crack-prone cultivars. But it is nevertheless clear that the data from peels and CM of Sausalito Cocktail are qualitatively intermediate between those from the crack-resistant and crack-prone cultivars (Fig. 2). The same trend was observed for the breaking stress (strength) and work of fracture (energy required for breaking), i.e., the peels and CM of the crack-resistant cultivar were mechanically more robust than those of the crack-prone cultivar, whereas Sausalito Cocktail peels were intermediate.

Comparisons among the moduli, breaking stresses, and works of fracture of peels versus their isolated $\mathrm{CM}$ indicated that the $\mathrm{CM}$ is substantially stiffer than its corresponding outer fruit wall for each cultivar. For example, the average Young's modulus of the crackresistant peels and their isolated CM are 44.2 and 71.2 MPa, respectively (Fig. 2). Although the breaking stresses of peels and their corresponding CM did not statistically differ significantly among the three cultivars, the work of fracture of isolated CM was significantly lower than that of their peels. This difference was attributed to the mechanical behavior of the fruit subepidermis, which is composed of collenchyma in all three cultivars.

Although the peels of all three cultivars consisted of a CM and underlying collenchyma (Fig. 3A-C), several anatomically significant differences among the cultivars were superimposed on this common groundplan. Specifically, the crack-resistant cultivar produced the thickest peels because of the greater combined depth of the CM and collenchyma (Fig. 3A-C). Cuticularization of cell walls extended two to three cell layers from the surface in the crack-resistant cultivar (Fig. 3A), to the inner

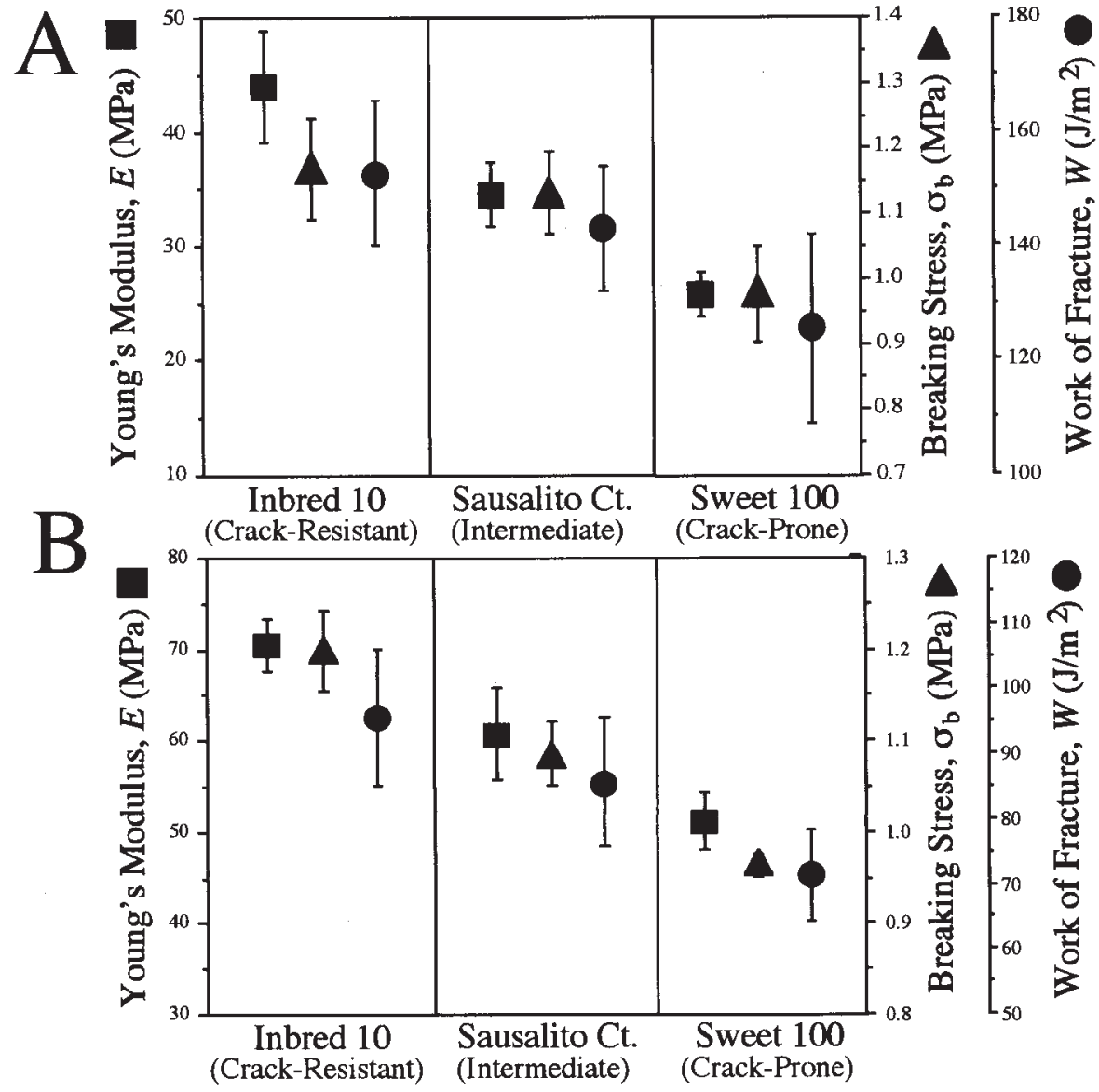

Fig. 2. Young's modulus (stiffness measured in tension), breaking stress (strength measured in tension), and work of fracture (energy required for breaking) of fruit peels (A) and isolated cuticular membranes (B). Means and se based on $n=10$. periclinal walls of the epidermis and to the anticlinal walls of the subepidermal layer in the intermediate cultivar (Fig. 3B), but only to the inner periclinal walls of the epidermis in the crack-prone cultivar (Fig. 3C). The collenchyma walls were thickest in the crack-resistant cultivar, thinnest in the crack-prone cultivar, and intermediate in thickness in the cultivar that occoasionally cracks (Fig. 3A-C). The outer periclinal cuticularized wall was thickest in the crack-resistant cultivar, thinnest in crackprone cultivar, and intermediate in thickness for the cultivar that occassionally cracks (Fig. $3 \mathrm{~A}-\mathrm{C})$. The anticlinal cuticularized walls of the epidermis were robustly thickened in the crack-resistant and intermediate cultivar and relatively uninterrupted by the pitting between epidermal cells (Fig. 3A, B, and D). In contrast, the anticlinal cuticularized walls of the epidermis in the crack-prone cultivar were tenuous to undetectable in the areas of pitting between epidermal cells (Fig. 3C).

In all three cultivars, the cuticularized walls of the CM were lined by cellulosic walls that resembled the walls of collenchyma in their optical properties. In the crack-resistant cultivar, these walls were virtually undetectable in unstained preparations with ordinary unpolarized illumination. They were revealed by staining to be nearly uniform in thickness within the epidermal and subepidermal cells, alike. In both of the other two cultivars, the cellulose wall layers were detected in unstained preparations (Fig. 3B-D) and in stained sections (Fig 3E), and were asymmetrically deposited within the epidermal cells. Examination of stained and unstained preparations with polarized light revealed that the net orientation of wall microfibrils in the cellulosic wall layers was parallel to the wall surfaces.

\section{Discussion}

The mechanical behavior and anatomy reported here for the fruit peels and their isolated $\mathrm{CM}$ are consistent with materials consisting of a viscoelastic matrix containing fibrillar components capable of passive realignment when placed in tension (Gordon, 1978; Köhler and Spatz, 2002; Niklas, 1992). Prior work indicates that tomato walls and their $\mathrm{CM}$ are viscoelastic (Matas et al., in press; Thompson, 2001), whereas the presence of fibrillar components in these biomaterials is substantiated by birefringent optical properties and strain-hardening behavior. We believe strain-hardening results from the alignment of fibrillar components in the direction of applied uniaxial tensile loads, whereas strain-softening is attributable to shearing failures at the interface of fibrils and their surrounding matrix, i.e., once aligned parallel to the direction of applied tensile loads, fibrils are expected to slip past one another as tensile loads exceed the breaking shear stress of the matrix in which fibrils are embedded.

However, the mechanical role of the fruit wall CM cannot be divorced from that of its underlying collenchyma, because this tissue is capable of substantial elastic deformation before breaking (Esau, 1936, 1977; Niklas, 


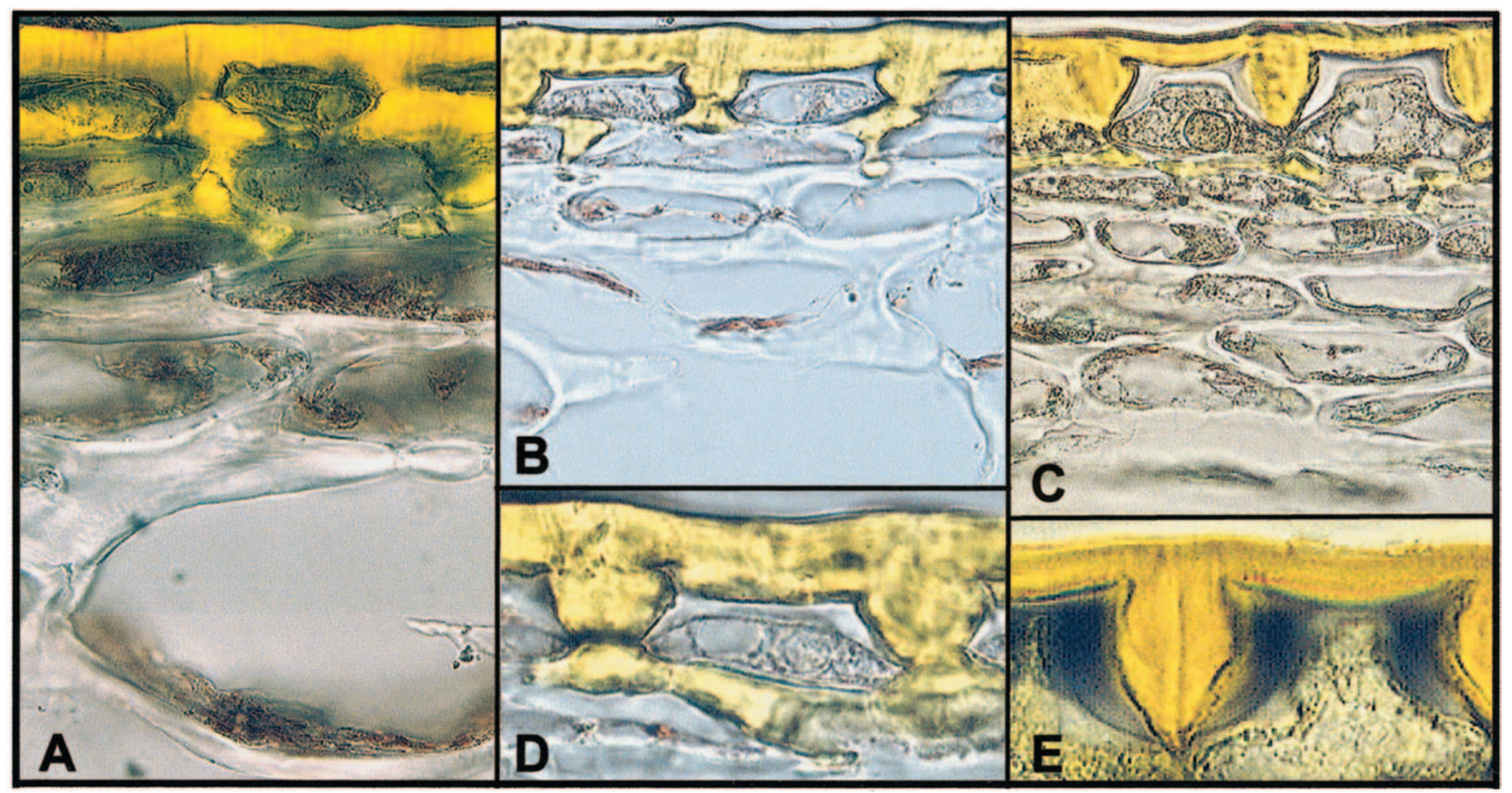

Fig. 3. Sections of outer fruit wall peels. (A) Unstained peel of Inbred 10 (crack-resistant); 570x. (B) Unstained peel of Sausalito Cocktail (crack-intermediate). 570x. (C) Unstained peel of Sweet 100 (crack-prone); 570x. Cuticularized walls have a yellow toorange color; collenchymatous walls appear grey to white in color. (D) Detail of epidermal cell of unstained Sausalito Cocktail peel. Note the inner periclinal wall is cuticularized in this example, as alternative to a collenchymatous wall. This feature is variable (see B for comparison). The thinnest part of the anticlinal walls is the location where pitting occurs between cells; $1040 \times$ (E) Peel of Sweet 100 stained with chlor-zinc-iodine. The cellulosic (collenchymatous) walls are stained dark grey and swollen due to contact with the staining reagent; $1700 \times$.

1992). The subepidermis thus undoubtedly serves as an energy sink that absorbs strain energy as it deforms and releases this energy when it elastically restores to its original dimensions. The presence of this sink is evident from comparisons between the works of fracture of fruit peels and their isolated CM. For each cultivar examined, the fruit wall's work of fracture is substantially greater than its corresponding $\mathrm{CM}$, which indicates that the subepidermis absorbs energy and extends considerably before breaking.

These results shed light on the phenomenology of tomato cracking. The CM of Inbred 10 (the crack-resistant cultivar) is thicker, stiffer, and stronger that that of Sweet 100 (the crackprone cultivar). It also has a higher work of fracture than the $\mathrm{CM}$ of either of the two other cultivars. Likewise, the CM of Sausalito Cocktail (the intermediate cultivar) has mechanical and anatomical properties that are more robust than those of the crack-prone cultivar. In this regard, it must be noted that no correlation is expected between the thickness of the CM and either its Young's modulus or breaking stress, because each of these two material properties is computed by normalizing the magnitudes of applied forces with respect to the cross sectional area of the CM. Therefore, the statistically significant differences observed among the Young's moduli and breaking stresses of the $\mathrm{CM}$ of the crack-resistant and crack-prone cultivars indicate that the $\mathrm{CM}$ of their fruit differs either in its material properties sensu stricto or in some microstructural aspect (e.g., the contiguity of the $\mathrm{CM}$ throughout subepidermal periclinal cell walls; see Fig. 3).
However, a strong correlation is expected between the thickness of the CM and the ability of the outer fruit wall to resist cracking, because the stiffness and strength of the pericarp are dependent on the quantity as well as the material properties of the CM (which has an average work of fracture comparable to that of polyesters; see Gordon, 1978). Therefore, even if the material properties of the CM are equivalent among cultivars, fruit with a thicker $\mathrm{CM}$ are expected to be more crack resistant than those with thinner CM. That the CM of the crack-resistant cultivar is thicker as well as stiffer and stronger than that of the crack-prone cultivar is thus expected.

The mechanism of cracking, however, remains problematic. The material properties of the CM or fruit wall provide only a measure of the ability of these structures to resist deformation when mechanically stressed. In contrast, the mechanism of cracking involves the modes of CM (and collenchyma) deformation and the genesis of stress. In this regard, we note that engineering theory predicts that the magnitudes of tensile stresses induced within the $\mathrm{CM}$ by turgor pressure will be equivalent in all directions provided that the fruit is perfectly spherical (Gordon, 1978). However, circumferential stresses will exceed longitudinal stresses for even slightly prolate fruit shapes, whereas longitudinal stresses will exceed circumferential stresses for even slightly oblate fruit shapes (Henry and Allen, 1974; Haman and Burgess, 1986). Thus, when the internal pressure within a fruit exceeds the yield stress of an isotropic CM, the CM is expected to rupture longitudinally or circum- ferentially in the case of prolate or oblate fruit, respectively. In this regard the fruit of all three cherry tomato cultivars are prolate and the fruit of the crack-prone and intermediate cultivars crack longitudinally without exception.

Because the yield stress of a CM can be lowered by pre- or post-harvest enzymatic processes, the external application of water, or an increase in relative humidity, the mechanisms responsible for fruit cracking are multifarious and likely to vary according to cultivar. Nevertheless, our data indicate that the thickness of the $\mathrm{CM}$ may provide a convenient and fairly reliable gauge of the propensity of fruit to crack, particularly in the absence of detailed biomechanical analyses. Such a quantitative character would be useful for QTL mapping of the genetic basis for resistance to cracking, although a complete understanding of cracking requires a QTL analysis of anatomical and mechanical factors over and above the thickness of the CM.

\section{Literature Cited}

Bennett, H.S. 1950. The microscopical investigation of biological materials with polarized light, $\mathrm{p}$. 591-677. In: M.R. McClung Jones (ed.). McClung's handbook of microscopical technique. Paul B. Hoeber, Inc., New York.

Cotner, S.D., E.E. Burns, and P.W. Leeper. 1969 Pericarp anatomy of crack-resistant and susceptible tomato fruit. J. Amer. Soc. Hort. Sci. 94:136-137.

Denny, M. W. 1988. Biology and the mechanics of the wave-swept environment. Princeton Univ. Press, Princeton, N.J.

Emmons, C.L.W. and J.W. Scott. 1997. Environmental and physiological effects on cuticle 
cracking in tomato. J. Amer. Soc. Hort. Sci. 122:797-801.

Emmons, C.L.W. and J.W. Scott. 1998. Ultrastructural and anatomical factors associated with resistance to cuticle cracking in tomato (Lycopersicon esculentum Mill.). Intl. J. Plt. Sci. 159:14-22.

Esau, K. 1936. Ontogeny and structure of collenchyma and of vascular tissues in celery petioles. Hilgardia 10:431-476.

Esau, K. 1977. Anatomy of seed plants. $2^{\text {nd }}$ ed. John Wiley and Sons, New York.

Gordon, J.E. 1978. Structures. Plenum Publ. Co., New York.

Haman, D.Z. and G.J. Burgess. 1986. Theoretical development for measuring the elastic properties of spherical cuticular membranes. Trans. Amer. Soc. Agr. Eng. 29:1470-1476.

Hankinson, B. and V.N.M. Rao. 1979. Histological and physical behaviour of tomato skins susceptible to cracking. Trans. Amer. Soc. Agr. Eng. 104:577-581.

Henry, Z.A. and W.H. Allen. 1974. Use of hydrostatic pressure in development of stress strain information for tomato skins. Trans. Amer. Soc. Agr. Eng. 99:787-792.

Holloway, P.J. 1982. Structure and histochemistry of plant cuticular membranes: An overview, p. 1-32. In: D.F. Cutler, K.L. Alvin, and C.E. Price (eds.). The plant cuticle. Academic Press,
London.

Jeffree, C. E. 1996. Structure and ontogeny of plant cuticles, p. 33-82. In: G. Kerstiens (ed.). Plant cuticles: An integrated functional approach. Bios Scientific Publ. Ltd., Oxford, U.K.

Köhler, L. and H.-C. Spatz. 2002. Micromechanics of plant tissues beyond the linear-elastic range. Planta 215:33-40.

Kutschera, U. 1989. Tissue stresses in growing plant organs. Physiol. Plantarum 77:157-163.

Matas, A.J., E.D. Cobb, J.A. Bartsch, D.J. Paolillo, Jr., and K.J. Niklas. 2004. Biomechanics and anatomy of Lycopersicon esculentum L. outer fruit walls and enzyme-treated samples. Amer. J. Bot. 91:352-360.

Niklas, K.J. 1992. Plant biomechanics. Univ. Chicago Press, Chicago, Ill.

Niklas, K.J. and D.J. Paolillo, Jr. 1997. The role of the epidermis as a stiffening agent in Tulipa (Liliaceae) stems. Amer. J. Bot. 84:735-744.

Niklas, K.J. and D.J. Paolillo, Jr. 1998. Preferential states of longitudinal tension in the outer tissues of Taraxacum officinale (Asteraceae) peduncles. Amer. J. Bot. 85:1068-1081.

Orgell, W.H. 1955. The isolation of plant cuticle with pectic enzymes. Plant Physiol. 30:78-80.

Peacock, H.D. 1966. Elementary microtechnique. E. Arnold, U.K.

Petracek, P.D. and M.J. Bukovac. 1995. Rheological properties of enzymatically isolated tomato fruit cuticle. Plant Physiology 109:675-679.

Roelofsen, P.A. 1959. The plant cell wall. Gebrüder Borntraeger, Berlin.

Round, A.N., B. Yan, S. Dang, R. Estephan, R.E. Stark, and J.D. Batteas. 2000. The influence of water on the nanomechanical behavior of the plant biopolyester cutin as studied by AFM and solid-state NMR. Biophysical J. 79:2761-2767.

Sekse, L. 1995. Fruit cracking in sweet cherries (Prunus avium L.). Some physiological aspectsA mini review. Scientia Hort. 63:135-141.

Thompson, D.S. 2001. Extensiometric determination of the rheological properties of the epidermis of growing tomato fruit. J. Expt. Bot. 52: 1291-1301.

Voisey, P.W., L.H. Lyall, and M. Kloek. 1970. Tomato skin strength - Its measurement and relation to cracking. J. Amer. Soc. Hort. Sci. 95:485-488.

Wattendorf, J. and P.J. Holloway. 1980. Studies on the ultrastructure and histochemistry of plant cuticles: the cuticular membrane of Agave americana L. in situ. Ann. Bot. 46:13-28.

Wiedermann, P. and C. Neinhuis. 1998. Biomechanics of isolated plant cuticles. Botanica Acta 111:28-34.

Yamada, Y., S.H. Wittwer, and M.J. Bukovac. 1964 Penetration of ions through isolated cuticles. Plant Physiol. 39:28-32. 